\title{
ON THE RENEWAL MEASURE FOR GAUSSIAN SEQUENCES
}

\author{
Fima C. KLEBANER \\ The University of Michigan, Department of Statistics. Ann Arbor. MI 48109-1027. USA \\ Received November 1985 \\ Revised March 1986
}

\begin{abstract}
A form for $U(t)$, the expected number of times a Gaussian sequence falls below a level $t$, is given in terms of the mean $M(x)$ and the variance $V^{2}(x)$ functions. It is shown that under general conditions $U(t)-M^{(-1)}(t), t \rightarrow \infty$. Moreover if $M$ and $V$ are regularly varying at infinity functions, then $U(t)-M^{(-1)}(t)$ is also regularly varying at infinity. A renewal theorem for stationary Gaussian sequences is given, where it is shown that the asymptotic behavior of $U(t)-t / \mu$ is determined by the asymptotic behavior of $V^{2}(t) / t$.
\end{abstract}

Keywords: renewal theorem. Gaussian sequence. stationary sequence.

\section{Introduction}

Let $\left\{\xi_{n}\right\}$ be a Gaussian sequence. Let $Y(A)=$ $\sum_{n=1}^{\infty} I\left(\xi_{n} \in A\right)$ be the number of visits to a Borel set $A$ by the process $\left\{\xi_{n}\right\}$; here $I(A)$ stands for the indicator of set $A$.

The expected number of visits to $A$ is the renewal measure of sequence $\left\{\xi_{n}\right\}$, which we denote by $U(A)$.

$U(A)=E Y(A)=\sum_{n=1}^{\infty} P\left(\xi_{n} \in A\right)$

Let $M_{n}, V_{n}^{2}$ denote the mean and the variance of $\xi_{n}$. Then $M$ and $V$ are positive functions from the set of integers into the reals.

The purpose of this note is to determine the behavior of $U$ on intervals $(-\infty, t]$ in terms of the functions $M, V$. The method presented here is simple and it allows one to determine the asymptotic behavior of $U$ from the asymptotic properties of the solution of a functional equation for $M$ and $V$ (equation (1) below).

The case when $\left\{\xi_{n}\right\}$ are cumulative sums of a

\footnotetext{
'Usually the renewal measure is defined as $U(A)+1$, where $U(A)$ is as above (cf. Feller, 1971).
}

stationary Gaussian sequence $\left\{X_{i}\right\}$ is of special interest. In a recent paper, Lalley (1985), showed that for an ergodic stationary sequence that satisfies the fading memory property a result similar to that of the classical renewal theory for independent random variables holds: $U(t+h)-U(t) \sim$ $h / \mu, t \rightarrow \infty$, where $U(t)=U((-\infty, t]), \mu=E X_{1}$ $>0$. As a corollary to the main result we obtain a renewal theorem for $\left\{\xi_{n}\right\}$. It is shown that if $\mu>0$ and $V$ is regularly varying at infinity with exponent $\alpha>\frac{1}{2}$ then $U(t)-t / \mu$ varies regularly at infinity with exponent $2 \alpha-1$. If $\alpha=\frac{1}{2}$ and $\lim _{t \rightarrow \infty} V^{2}(t) / t=\infty$ then

$U(t)-t / \mu \sim V^{2}(t) / 2 \mu^{2} t, \quad t \rightarrow \infty$.

If $\lim _{t \rightarrow \infty} V^{2}(t) / t=\sigma^{2}<\infty$ then

$U(t)-t / \mu=\sigma^{2} / 2 \mu^{2}-r_{t}+o(1)$

where $0<r_{t}<1$. It is also conjectured that in the latter case $\lim _{t \rightarrow \infty} r_{t}=\frac{1}{2}$. For the comparison with the above results we state a renewal theorem for sums of independent and identically distributed sequences $\left\{X_{i}\right\}$. If $\mu=E\left(X_{1}\right)>0$ and $\sigma^{2}=$ $\operatorname{Var}\left(X_{1}\right)<\infty$ then

$$
U(t)-t / \mu=\sigma^{2} / 2 \mu^{2}-\frac{1}{2}+\mathrm{o}(1), \quad t \rightarrow \infty
$$

(Feller, 1971, p. 387). If, however, $\sigma^{2}=\infty$ then 
$U(t)-t / \mu$ can vary regularly at infinity (Feller, 1971, p. 373).

I also conjecture that the results obtained for stationary Gaussian sequences will also hold for other stationary sequences when the assumption of normality is dropped.

\section{Notations and assumptions}

Let $M(x)$ and $V^{2}(x)$ be positive continuous functions defined for all $x \geqslant 1$ such that $M(x)=$ $M_{n}, V^{2}(x)=V_{n}^{2}$ when $x$ takes an integer value $n$. We shall assume that $M, V$ are positive and increasing functions. Let $t \geqslant 0$ and $U(t)=$ $U((-\infty, t])$. It is easy to see that for $U(t)$ to be finite it is necessary that $M(x) / V(x) \rightarrow \infty, x \rightarrow$ $\infty$. Therefore, it will be assumed that $V(x)=$ o $(M(x)), x \rightarrow \infty . M(x) / V(x)$ tends to $+\infty$, we shall assume that it is increasing. Hence there exists the inverse of $M(x) / V(x)$ which we denote by $R(x) . R(x)$ is positive, continuous and is increasing to $+\infty$. Denote by $W(x)$ the inverse function of $M(x)$.

Define for $x \geqslant 1, h_{t}(x)=(t-M(x) / V(x)$, we shall assume that, for any $t \geqslant 0, h_{t}(x)$ is decreasing in $x$. Then there exists the inverse function $g_{t}(x) . g_{t}(x)>0, g_{t}(x)$ is decreasing and it maps $\left(-\infty, h_{t}(1)\right]$ onto $[1,+\infty)$. We extend the definition of $g_{t}(x)$ to the whole line and define it to be zero for $x>h_{t}(1)$. With a slight abuse of notations we shall call the new function also $g_{t}(x)$. For $x \geqslant 0,[x],\{x\}$ will denote respectively the integer and the fractional part of $x$.

\section{Results}

Proposition 1. $U(t)=E\left(\left[g_{t}(Z)\right]\right)$, where $Z$ $N(0,1)$ is the standard normal random variable and $g_{t}(x)$ is the solution of

$M\left(g_{t}(x)\right)=t-x V\left(g_{t}(x)\right), \quad x \in\left(-\infty, h_{t}(1)\right]$.

Corollary 1. $U(t)=E\left(g_{t}(z)\right)-r_{t}$, where $0<r_{t}<1$.

Proposition 2. Assume that $R(x)$ satisfies

$$
\lim _{x \rightarrow \infty} R(x+1) / R(x)=1 \text {; }
$$

there exists a constant $C$ such that, for all $x, y \geqslant 1$,

$R(x+y) \leqslant C R(x) R(y)$.

Then

$U(t) \sim W(t), \quad t \rightarrow \infty$.

Remark 1. The assumption that $V(x)$ is increasing is not used in Proposition 2. Renewal theory for sequences of the form

$\xi_{n}=\left(X_{1}+X_{2}+\cdots+X_{n}\right) / \sqrt{n}$,

where $X_{1}, X_{2}, \ldots$ are i.i.d. Gaussian with positive expectation, is of considerable interest in sequential analysis (cf. Woodroofe, 1976; also Lai and Siegmund, 1977).

For the sequences $\left\{\xi_{n}\right\}$ mentioned above Proposition 2 gives $U(t)-t^{2} / \mu^{2}, t \rightarrow \infty$, where $\mu=$ $E\left(X_{1}\right)$. It is not hard to deduce from Proposition 1 and its Corollary that $U(t)=t^{2} / \mu^{2}+\sigma^{2} / \mu^{2}+r_{t}+$ $o(1), t \rightarrow \infty$, where $\sigma^{2}=\operatorname{Var}\left(X_{1}\right)$ and $0<r_{t}<1$. Woodroofe gives this expansion down to the terms o(1).

The main result is given in Proposition 3 and its Corollaries.

Proposition 3. Let $M, V$ be increasing, regularly varying at infinity functions with exponents $\beta$ and $\alpha$ respectively. $\beta>\alpha>0$. Suppose also $M^{\prime}, M^{\prime \prime}, V^{\prime}$ exist and are monotone for large values of $x$. Then

$$
\begin{aligned}
& E\left(g_{t}(Z)\right)-W(t) \\
& \quad-(1+2 \alpha-\beta) W(t) V^{2}(W(t)) / 2 \beta^{2} t^{2}, t \rightarrow \infty .
\end{aligned}
$$

Corollary 2. Let $M$ and $V$ be as in Proposition 3. Suppose also that

$W(t) V^{2}(W(t)) / t^{2} \rightarrow \infty \quad$ as $t \rightarrow \infty$.

Then

$$
\begin{aligned}
& U(t)-W(t) \\
& \quad \sim(1+2 \alpha-\beta) W(t) V^{2}(W(t)) / 2 \beta^{2} t^{2}, t \rightarrow \infty .
\end{aligned}
$$

Renewal theory for sums of a stationary, Gaussian sequence is of special interest. In this case the above results imply

Corollary 3 (A renewal theorem for stationary Gaussian sequences). Let $\xi_{n}+X_{1}+X_{2}+\cdots+X_{n}$ 
$+n \mu$, where $X_{1}, X_{2}, \ldots$ is a stationary Gaussian sequence with $E\left(X_{1}\right)=0$ and $\mu>0$. Suppose $V_{n}^{2}=$ $\operatorname{Var}\left(\xi_{n}\right)$ is regularly varying at infinity with exponent $2 \alpha$. (i) If $\alpha>\frac{1}{2}$ or $\alpha=\frac{1}{2}$ and $\lim _{t \rightarrow \infty} V^{2}(t) / t$ $=+\infty$ then

$U(t)-t / \mu \sim \alpha V^{2}(t) / \mu^{2} t, \quad t \rightarrow \infty$.

(ii) If $\alpha=\frac{1}{2}$ and $\lim _{t \rightarrow \infty} V^{2}(t) / t=\sigma^{2}, 0 \leqslant \sigma^{2}$ $<\infty$, then

$$
\begin{aligned}
\frac{\sigma^{2}}{2 \mu^{2}}-1 & \leqslant \lim _{t \rightarrow \infty} \inf (U(t)-t / \mu) \\
& \leqslant \lim _{t \rightarrow \infty} \sup (U(t)-t / \mu) \leqslant \frac{\sigma^{2}}{2 \mu^{2}} .
\end{aligned}
$$

Remark 2. The case (ii) describes the behavior of the renewal measure of the sums of stationary mixing Gaussian variables with positive mean $\mu$. In order to establish existence of $\lim _{t \rightarrow \infty}(U(t)-$ $t / \mu$ ) we have to show the existence of $\lim _{t \rightarrow \infty} r_{t}$, which I am not able to do at this stage. I, however, conjecture that $\lim _{t \rightarrow \infty} r_{t}$ exists and is equal to $\frac{1}{2}$. If this is the case then the renewal theorem for stationary mixing Gaussian sequences takes the same form as the classical renewal theorem for i.i.d. random variables except for the fact that instead of being $\operatorname{Var}\left(X_{1}\right), \sigma^{2}$ stands for the $\lim _{n \rightarrow \infty}\left(\operatorname{Var}\left(\sum_{i=1}^{n} X_{i}\right)\right) / n$.

\section{Proofs}

In proofs the following properties of regularly varying functions are used. Let $f(x)$ be an increasing, regularly varying at infinity with exponent $\lambda$ function.

(P1) The inverse function of $f(x)$ is regularly varying at infinity with exponent $1 / \lambda$.

(P2) If $f^{\prime}(x)$ exists and is monotone for large $x$, then $x f^{\prime}(x) \sim \lambda f(x), x \rightarrow \infty$.

(P3) If $f_{1}(x) \sim f_{2}(x) \rightarrow \infty, \quad x \rightarrow \infty$, then $f\left(f_{1}(x)\right)-f\left(f_{2}(x)\right), x \rightarrow \infty$.

The proofs of (P1) and (P2) may be found in the book of Seneta. (P3) is an immediate consequence of monotonicity of $f$ and regular variation.

\section{Proof of Proposition 1}

$U(t)=\sum_{n=1}^{\infty} P\left(\xi_{n} \leqslant t\right)=\sum_{n=1}^{\infty} P\left(Z \leqslant h_{t}(n)\right)$

$$
\begin{aligned}
& =\sum_{n=1}^{\infty} E\left(I\left(h_{t}(n) \geqslant Z\right)\right) \\
& =E\left(\sum_{n=1}^{\infty} I\left(h_{t}(n) \geqslant Z\right)\right) \\
& =E\left(\sum_{n=1}^{\infty} I\left(n \leqslant g_{\imath}(Z)\right)\right)=E\left(\left[g_{t}(Z)\right]\right) .
\end{aligned}
$$

$h_{t}\left(g_{t}(x)\right)=x$ for $x \leqslant h_{t}(1)$, therefore (1) holds.

Proof of Corollary 1. $r_{t}=E\left(\left\{g_{t}(Z)\right\}\right)$.

Proof of Proposition 2. We first show that, for any fixed $x \in(-\infty, \infty)$,

$g_{t}(x) \sim W(t), \quad t \rightarrow \infty$.

To this end, write $g_{t}(x)=R\left(t / V\left(g_{t}(x)\right)-x\right)$, which follows from (1). Since $g_{t}(x)$ is decreasing, $V(x)$ is nondecreasing and $R(x)$ is increasing, we obtain the following inequality for any $x<h_{r}(1)$ :

$R\left(\tau-x^{+}\right)<g_{t}(x)<R\left(\tau+x^{-}\right)$,

with $\tau=t / V\left(g_{1}(0)\right)$ and $x^{+}, x^{-}$being standard notations, $x^{+}=\max (x, 0), x^{-}=\max (-x, 0)$. Notice that

$W(t)=g_{t}(0)=R(\tau)$,

and that $\tau=t / V\left(g_{t}(0)\right)=M(W(t) / V(W(t))$. Hence $\tau \rightarrow \infty$ as $t \rightarrow \infty$. (2) now follows from (3) by using (A1). Since $h_{t}(1) \rightarrow \infty$, as $t \rightarrow \infty$, (2) holds for any $x$.

Using (A2) and monotonicity of $R(x)$ it is not hard to establish that there exists a constant $C_{1}$ such that, for' all $x \in(-\infty, \infty)$,

$\left|g_{t}(x)-W(t)\right| \leqslant C_{1} R(|x|) \cdot W(t)$,

Since $R(x)$ is continuous, it follows from (A1) that $R(x)$ must be of the form $\mathrm{e}^{\rho(x)}$, where $\rho(x)$ $=\mathrm{o}(x), x \rightarrow \infty$ (Seneta, 1976, p. 30). Therefore, $E(R(|Z|))<\infty . \quad E\left(g_{t}(Z)\right) \sim W(t), \quad t \rightarrow \infty$, now follows by the dominated convergence from (2) and (4). The result follows from Corollary 1 , since $W(t) \rightarrow \infty$ as $t \rightarrow \infty$.

Proof of Proposition 3. We show first that, for any $x$,

$g_{t}(x)-W(t) \sim-x W^{\prime}(t) \cdot V(W(t)), \quad t \rightarrow \infty$. 
Taking Taylor expansion of $W$ at $t$ we obtain, from (1),

$g_{t}(x)-W(t)=-x W^{\prime}\left(\theta_{t}\right) \cdot V\left(g_{t}(x)\right)$,

$\theta t \in\left(t, M\left(g_{t}(x)\right)\right.$.

Taking the Taylor expansion of $V$ at $g_{t}(0)$,

$$
\begin{aligned}
& g_{t}(x)-W(t)=-x W^{\prime}\left(\theta_{t}\right) V(W(t)) \\
&-x W^{\prime}\left(\theta_{t}\right) V^{\prime}\left(\zeta_{t}\right)\left(g_{t}(x)-W(t)\right), \\
& \zeta_{t} \in\left(g_{t}(0), g_{t}(x)\right) .
\end{aligned}
$$

From (7) we obtain

$$
\frac{\left(g_{t}(x)-W(t)\right)}{\left(W^{\prime}(t) V(W(t))\right.}=\frac{-x}{1+x W^{\prime}\left(\theta_{t}\right) V^{\prime}\left(\zeta_{t}\right)} \frac{W^{\prime}\left(\theta_{t}\right)}{W^{\prime}(t)} .
$$

Using (P1) and (P2) one can see that $W, R, W^{\prime}$, $V^{\prime}$ are regularly varying at $\infty$ functions. It can also be seen that $R$ is regularly varying at $\infty$ and it satisfies (A1) and (A2). From (2) we obtain $\theta_{t}-t$ and $\zeta_{t} \sim W(t)$ as $t \rightarrow \infty$. Hence $W^{\prime}\left(\theta_{t}\right) \sim$ $W^{\prime}(t), \quad V^{\prime}\left(\zeta_{t}\right) \sim V^{\prime}(W(t))$ and $W^{\prime}\left(\theta_{t}\right) \cdot V^{\prime}\left(\zeta_{t}\right) \rightarrow 0$ as $t \rightarrow \infty$. (5) now follows from (8).

Taking one more term in the Taylor expansion of $W$ we obtain

$$
\begin{aligned}
g_{t}(x)-W(t)= & -x W^{\prime}(t) V(W(t)) \\
& -x W^{\prime}(t) V^{\prime}\left(\zeta_{t}\right)\left(g_{t}(x)-W(t)\right) \\
& +x^{2} / 2 W^{\prime \prime}\left(\eta_{t}\right) V^{2}\left(g_{t}(x)\right) \\
= & -x W^{\prime}(t) V(W(t))+T_{t}^{1}(x) \\
& +T_{t}^{2}(x)
\end{aligned}
$$

where $\eta_{t} \in\left(t, M\left(g_{t}(x)\right)\right)=\left(t, t-x V\left(g_{t}(x)\right)\right)$. Using (5), (P2), (P3), and $\zeta_{t} \sim W(t), \eta_{n}-t$ as $t \rightarrow \infty$ we obtain that

$T_{t}^{l}(x) \sim \alpha x^{2} W(t) V^{2}(W(t)) / \beta^{2} t^{2}, \quad t \rightarrow \infty$.

and

$T_{t}^{2}(x) \sim-(\beta-1) x^{2} W(t) V^{2}(W(t)) / 2 \beta^{2} t^{2}$.

$$
t \rightarrow \infty \text {. }
$$

We wish to replace $x$ by $Z$ in (9), take expectations and pass to the limit as $t \rightarrow \infty$. Therefore, we shall construct integrable bounds for $\left|T_{t}^{i}(Z)\right|$, $i=1,2$. We show that there exist constants $C_{2}>0$, $\gamma>0$ such that, for all $x<h_{t}(1)$,

$$
\begin{aligned}
& \left|T_{t}^{i}(x)\right| \cdot t^{2} / W(t) V^{2}(W(t))<C_{2} R^{\gamma}(|x|), \\
& \quad i=1,2 .
\end{aligned}
$$

Since the value of constants is not important and there are many different constants to be used in the following inequalities, we shall drop the subscripts. In what follows $C$ will denote some positive finite constant, which may be different if used in different inequalities.

It can be seen from (2) and (A2) that the following inequalities hold.

$1<g_{t}(x) / g_{t}(0)<C R(|x|), \quad x<0$,

and

$(C R(x))^{-1}<g_{t}(x) / g_{i}(0)<1, \quad 0<x<h_{t}(1)$.

$M(x) x^{-(\beta+1)}$ is decreasing for large $x$. It follows that, for all $0<x<h_{i}(1)$

$M\left(g_{t}(x)\right) / t>C R^{-(\beta+1)}(x)$.

(14) and $M\left(g_{t}(0)\right)=t$ were used to establish (15). $V(x) x^{-(\alpha+1)}$ is decreasing for large $x$. It follows that, for all $x<0$,

$V\left(g_{t}(x)\right) / V\left(g_{i}(0)<C R^{\alpha+1}(|x|)\right.$.

(13) was used to establish (16).

Consider $T_{t}^{1}$. By $(\mathrm{P} 2), \quad V^{\prime}\left(\zeta_{t}\right) \leqslant C V\left(\zeta_{t}\right) / \zeta_{t}$, $W^{\prime}\left(\theta_{t}\right) \leqslant C W\left(\theta_{t}\right) / \theta_{t}$ for some $C>0$. This together with (6) gives

$$
\frac{\left|T_{t}^{1}(x)\right| t^{2}}{W(t) V^{2}(W(t))} \leqslant \frac{C^{2} x^{2} V\left(\zeta_{t}\right) W\left(\theta_{t}\right) t}{\zeta_{t} \theta_{t} V^{2}(W(t))} .
$$

If $x>0$ then $M\left(g_{t}(x)\right)<\theta_{t}<t, g_{t}(x)<\zeta_{t}<g_{t}(0)$ $=W(t)$ and, by monotonicity of $V$ and $W$, (17) does not exceed

$C^{2} x^{2}\left(\left(M\left(g_{t}(x) / t\right)\left(g_{t}(x) / g_{t}(0)\right)^{-1}\right.\right.$.

Using (14) and (15) we obtain that an upper bound for the expression in (17) when $x>0$ is given by $C x^{2} R^{\beta+2}(x)$.

If $x<0$ then $t<\theta_{t}<M\left(g_{t}(x)\right), g_{t}(0)<\zeta_{t}<$ $g_{r}(x)$ and, by monotonicity of $V$ and $W,(17)$ does 
not exceed

$C x^{2}\left(g_{t}(x) / g_{t}(0)\right)\left(V^{2}\left(g_{t}(x) / V^{2}\left(g_{t}(0)\right)\right)\right.$.

Using (13) and (16) we obtain that an upper bound for the expression in (17) when $x<0$ is given by $C x^{2} R^{2 \alpha+3}(|x|)$.

Take $\gamma=\max (2 \alpha+3,2 \beta+2)$. Thus we have established (12) for $T_{1}^{1}$.

Consider $T_{r}^{2}$. Using an upper bound for $W^{\prime \prime}$ from (P2), we can see that

$$
\frac{\left|T_{t}^{2}(x)\right| t^{2}}{W(t) V^{2}(W(t))} \leqslant C x^{2} \frac{W\left(\eta_{t}\right) V^{2}\left(g_{t}(x)\right) t^{2}}{\eta_{t}^{2} W(t) V^{2}(W(t))} .
$$

If $x>0, M\left(g_{t}(x)\right)<\eta_{t}<t, g_{t}(x)<g_{t}(0)$. Hence the expression in (18) does not exceed

$C\left(t / M\left(g_{t}(x)\right)^{2}\right)<C x^{2} R^{2 \beta+2}(x)$,

the last inequality by using (15). If $x<0, t<\eta_{t}<$ $M\left(g_{t}(x)\right), g_{r}(x)>g_{r}(0)$. Hence the expression in $(18)$ is less than

$$
\begin{aligned}
& C x^{2}\left(g_{t}(x) / g_{t}(0)\right)\left(V\left(g_{t}(x)\right) / V\left(g_{t}(0)\right)\right)^{2} \\
& \quad<C x^{2} R^{2 \alpha+3}(|x|),
\end{aligned}
$$

the last inequality by (13) and (16). Thus we have established (12) for $T_{t}^{2}$.

Hence from (10), (11) and (12) we obtain

$$
\begin{aligned}
& E\left(\left(T_{t}^{1}(Z)+T_{t}^{2}(Z)\right) I\left(Z<h_{t}(1)\right)\right) \\
& \sim(1+2 \alpha-\beta) W(t) V^{2}(W(t)) / 2 \beta^{2} t^{2}, \\
& \quad t \rightarrow \infty .
\end{aligned}
$$

Let $x$ be $Z$ in (9) and take expectations to obtain

$$
\begin{aligned}
E\left(g_{t}(Z)\right)-W(t) & \\
= & W^{\prime}(t) V\left(W(t) E\left(Z I\left(Z>h_{t}(1)\right)\right)\right) \\
& \quad+E\left(T_{t}^{\mathrm{l}}(Z)+T_{t}^{2}(Z) I\left(Z<h_{t}(1)\right) .\right.
\end{aligned}
$$

Since $E\left(Z I\left(Z>h_{t}(1)\right)\right)=(1 / \sqrt{2 \pi}) \mathrm{e}^{-h_{t}(1)}$, the term $W^{\prime}(t) V(W(t)) \mathrm{e}^{-h_{,}(1)}=\mathrm{o}(1)$ as $t \rightarrow \infty$. The Proposition now follows from (20) and (19).

Proof of Corollary 2 follows from Proposition 3 and Corollary 1.

Proof of Corollary 3. (i) follows from Corollary 2 and properties of regular variation of $V$.

(ii) Proposition 3 yields

$$
E\left(g_{t}(Z)\right)-t / \mu-\frac{\sigma^{2}}{2 \mu^{2}}, \quad t \rightarrow \infty .
$$

The result follows from Corollary 1.

\section{Acknowledgement}

The author is thankful to the referee for the content of Remark 1 and other valuable comments, which improved the original version of the paper.

\section{References}

Feller, W. (1971), An Introduction to Probability Theory and its Applications - II (John Wiley and Sons, New York).

Lalley, S. (1985), A renewal theorem for a class of stationary sequences. Preprint.

Lai, T.L. and D. Siegmund (1977), A nonlinear renewal theory with applications to sequential analysis I. The Annals of Statistics 5 (5), 946-954.

Seneta, E. (1976). Regularly Varying Functions (Springer, Lecture Notes in Mathematics, New York).

Woodroofe, M. (1976), A renewal theorem for curved boundaries and moments of first passage times, The Annals of Probability 4 (1), 67-80. 